\title{
Three-dimensional cascaded lattice Boltzmann method: Improved implementation and consistent forcing scheme
}

\author{
Linlin Fei, ${ }^{1}$ Kai H. Luo, ${ }^{1,2, *}$ and Qing $\mathrm{Li}^{3, \dagger}$ \\ ${ }^{1}$ Center for Combustion Energy; Key laboratory for Thermal Science and Power Engineering of Ministry of Education, \\ Department of Energy and Power Engineering, Tsinghua University, Beijing 100084, China \\ ${ }^{2}$ Department of Mechanical Engineering, University College London, Torrington Place, London WC1E 7JE, UK \\ ${ }^{3}$ School of Energy Science and Engineering, Central South University, Changsha 410083, China
}

(Received 22 January 2018; published 25 May 2018)

\begin{abstract}
The cascaded or central-moment-based lattice Boltzmann method (CLBM) proposed in [Phys. Rev. E 73, 066705 (2006)] possesses very good numerical stability. However, two constraints exist in three-dimensional (3D) CLBM simulations. First, the conventional implementation for 3D CLBM involves cumbersome operations and requires much higher computational cost compared to the single-relaxation-time (SRT) LBM. Second, it is a challenge to accurately incorporate a general force field into the 3D CLBM. In this paper, we present an improved method to implement CLBM in 3D. The main strategy is to adopt a simplified central moment set and carry out the central-moment-based collision operator based on a general multi-relaxation-time (GMRT) framework. Next, the recently proposed consistent forcing scheme for CLBM [Fei and Luo, Phys. Rev. E 96, 053307 (2017)] is extended to incorporate a general force field into 3D CLBM. Compared with the recently developed nonorthogonal CLBM [Rosis, Phys. Rev. E 95, 013310 (2017)], our implementation is proved to reduce the computational cost significantly. The inconsistency of adopting the discrete equilibrium distribution functions in the nonorthogonal CLBM is analyzed and validated. The 3D CLBM developed here in conjunction with the consistent forcing scheme is verified through numerical simulations of several canonical force-driven flows, highlighting very good properties in terms of accuracy, convergence, and consistency with the nonslip rule. Finally, the techniques developed here for 3D CLBM can be applied to make the implementation and execution of 3D MRT-LBM more efficient.
\end{abstract}

DOI: 10.1103/PhysRevE.97.053309

\section{INTRODUCTION}

As a mesoscopic numerical method based on the kinetic theory, the lattice Boltzmann method (LBM) has gained remarkable success for the simulation of complex fluid flows and beyond during the past three decades [1-15]. Different from the conventional computational fluid dynamics (CFD) methods where the macroscopic governing equations are solved numerically, LBM solves a discrete Boltzmann equation which is designed to reproduce the Navier-Stokes (N-S) equations in the macroscopic limit. In the LBM simulation, the fluid is usually represented by populations of fictitious particles colliding locally and streaming to adjacent nodes along the links of a regular lattice. The scale-bridging nature of LBM allows its natural incorporation of microscopic and/or mesoscopic physics, while the highly efficient collision-streaming algorithm makes it affordable computationally [10].

In the practical implementation, the simplest approach to represent particles colliding process is to relax all the distri-

\footnotetext{
*Corresponding author: K.Luo@ucl.ac.uk

†Corresponding author: qingli@csu.edu.cn
}

Published by the American Physical Society under the terms of the Creative Commons Attribution 4.0 International license. Further distribution of this work must maintain attribution to the author $(s)$ and the published article's title, journal citation, and DOI. bution functions (DFs) to their local equilibria at an identical rate, which is known as the single-relaxation-time (SRT) model [16]. While the SRT-LBM is successful in many fluid flows, it may suffer numerical instability for flows with relatively low viscosities [17,18], as well as inaccuracy in implementing the boundary conditions $[19,20]$. Compared with the SRT model, the multiple-relaxation-time (MRT) model, where the collision step is carried out in the raw moment space, is able to enhance the stability of LBM by carefully separating the timescales among the kinetic modes $[17,18,21]$. In addition, the MRTLBM can also improve the numerical accuracy for nonslip boundary conditions by adopting a so-called magic parameter $[19,20]$. In 2006, a cascaded collision operator was proposed by Geier et al. [22], where the collision step is implemented in the central moment space. To get the higher-order postcollision central moments, the lower-order ones are needed, which implies, from the lowest order to the highest order, i.e., a cascaded operation procedure. The cascaded lattice Boltzmann method (CLBM) increases the numerical stability significantly, which is also essentially due to the removal of the ghost modes. Besides, relaxation in a co-moving frame of reference, i.e., in terms of central moments, allows a natural setting to achieve better Galilean invariance, compared with relaxation in the frame at rest, for a specified discrete velocity set [22]. More comparisons and discussions between relaxations in the raw moment and central moment spaces can be found in Refs. [22-26]. 
More recently, many studies have been carried out to improve the cascaded collision model and apply the CLBM to practical applications. In Ref. [27], Asinari argued that CLBM essentially consists in using a generalized local equilibrium in the frame at rest. In addition, a multiphase CLBM has been developed to simulate multiphase flows by LycettBrown and Luo $[28,29]$. They further extended the model with an improved forcing scheme for large density ratio multiphase flows at high Reynolds and Weber numbers [30]. Moreover, a thermal cascaded LBM (TCLBM) has been proposed by the present authors to simulate low-Mach compressible thermal flows [31], and several different CLBMs have been developed later for incompressible thermal flows [32-36]. Finally, CLBM has also been extended to simulate shallow water equations [37], moving boundary problems [38], as well as stationary flows with a preconditioning method [39].

Although the CLBM possesses very good numerical stability and has achieved success for a series of complex flows, two critical problems still exist in the three-dimensional (3D) simulations. First, the practical implementation for 3D CLBM in the original work [22] involves a lot of cumbersome notations and the computational cost is much higher than the SRT-LBM. Even if some efforts have been made [24,29,40], it is still quite difficult to handle the expressions compared with the SRT-LBM. For example, a naive implementation of the most recent nonorthogonal CLBM [40] needs a CPU time that is much larger than that by the SRT-LBM. Second, an accurate and easy-to-implement forcing scheme is needed to incorporate an external or internal force field into the 3D CLBM. In 2009, Premnath et al. proposed a forcing scheme for CLBM by method of central moments [23], which was then extended to 3D model in Ref. [24]. In Refs. [28-30], the SRT-style forcing scheme was used in the CLBM. Besides, an alternative forcing scheme based on a discrete equilibrium has been developed by De Rosis [41]. Recently, we proposed an alternative derivation for the CLBM by introducing a shift matrix, where the shift matrix is used to shift raw moments to be central moments [42]. This approach puts the MRT-LBM and CLBM into a general multi-relaxation-time (GMRT) framework and clarifies the relationship between them. Based on this GMRT framework, we proposed a consistent forcing scheme to incorporate a general time and space-varying force field into CLBM, where "consistent" means that the forcing scheme can degrade into the widely used forcing scheme in MRT-LBM [43] and SRT-LBM [44] under the corresponding conditions when CLBM degrades into MRT-LBM and SRT-LBM, respectively. Compared with the forcing schemes in Refs. [23,28,41], the consistent forcing scheme has better numerical performances in terms of accuracy, isotropy, and faithful implementation of the nonslip boundary condition. Therefore, the present paper aims to simplify the implementation and reduce the computational cost for 3D CLBM. In the meantime, the consistent forcing scheme is extended to incorporate a general force field for 3D flows.

The rest of this paper is organized as follows. Section II presents the improved implementation and the consistent forcing scheme for 3D CLBM. Numerical verifications are carried out in Sec. III. Finally, concluding remarks are given in Sec. IV.

\section{IMPROVED 3D CLBM WITH CONSISTENT FORCING SCHEME}

The improved implementation is based on the GMRT framework and adopts a new central moment set. First, the GMRT framework with the consistent forcing scheme is introduced briefly. Then, the choices of the central moment set, central moment equilibria, and forcing terms in the central moment space are given in detail.

\section{A. GMRT framework}

In the present work, we focus on the standard D3Q27 discrete velocity model (DVM). However, it should be noted that the procedures shown in this work are not limited to the specified DVM and can be extended to other DVMs readily. For example, a D3Q19 CLBM can be directly extracted from the D3Q27 CLBM (see in the Appendix A). The lattice speed $c=\Delta x / \Delta t=1$ and the lattice sound speed $c_{s}=1 / \sqrt{3}$ are adopted, in which $\Delta x$ and $\Delta t$ are the lattice spacing and time step. The discrete velocities $\mathbf{e}_{i}=\left[\left|e_{i x}\right\rangle,\left|e_{i y}\right\rangle,\left|e_{i z}\right\rangle\right]$ are defined as

$$
\begin{gathered}
\left|e_{i x}\right\rangle=[0,1,-1,0,0,0,0,1,-1,1,-1,1,-1,1, \\
-1,0,0,0,0,1,-1,1,-1,1,-1,1,-1]^{\top}, \\
\left|e_{i y}\right\rangle=[0,0,0,1,-1,0,0,1,1,-1,-1,0,0,0,0,1, \\
-1,1,-1,1,1,-1,-1,1,1,-1,-1]^{\top}, \\
\left|e_{i z}\right\rangle=[0,0,0,0,0,1,-1,0,0,0,0,1,1,-1,-1,1,1, \\
-1,-1,1,1,1,1,-1,-1,-1,-1]^{\top},
\end{gathered}
$$

where $i=0,1, \ldots, 26,|\cdot\rangle$ denotes a 27 -dimensional column vector, and the superscript $T$ denotes the transposition.

We first define the raw and central moments of the discrete distribution functions (DFs) $f_{i}$,

$$
\begin{aligned}
& k_{m n p}=\left\langle f_{i} \mid e_{i x}^{m} e_{i y}^{n} e_{i z}^{p}\right\rangle, \\
& \tilde{k}_{m n p}=\left\langle f_{i} \mid\left(e_{i x}-u_{x}\right)^{m}\left(e_{i y}-u_{y}\right)^{n}\left(e_{i y}-u_{z}\right)^{p}\right\rangle,
\end{aligned}
$$

where $m, n$, and $p$ are integers and $u_{x}, u_{y}$, and $u_{z}$ are velocity components in the $x, y$, and $z$ directions, respectively. The equilibrium values $k_{m n p}^{\mathrm{eq}}$ and $\tilde{k}_{m n p}^{\mathrm{eq}}$ are defined analogously by replacing $f_{i}$ with the discrete equilibrium distribution functions (EDFs) $f_{i}^{\mathrm{eq}}$. To construct the central-moment-based collision operator, a raw moment set $\left|T_{i}\right\rangle$ and the corresponding central moment set $\left|\tilde{T}_{i}\right\rangle$ are needed,

$$
\left|T_{i}\right\rangle=\left[T_{0}, T_{1}, \ldots, T_{26}\right]^{\top}, \quad\left|\tilde{T}_{i}\right\rangle=\left[\tilde{T}_{0}, \tilde{T}_{1}, \ldots, \tilde{T}_{26}\right]^{\top},
$$

where the elements in $\left|T_{i}\right\rangle$ and $\left|\tilde{T}_{i}\right\rangle$ are combinations of $k_{m n p}$ and $\tilde{k}_{m n p}$ in the ascending order of $(m+n+p)$, respectively. According to Eq. (2), the transformation from the discrete DFs to their raw moments can be performed through a transformation matrix $\mathbf{M}$, and the shift from the raw moments to central moments can be performed through a shift matrix $\mathbf{N}$,

$$
\left|\tilde{T}_{i}\right\rangle=\mathbf{N}\left|T_{i}\right\rangle=\mathbf{N M}\left|f_{i}\right\rangle .
$$

The explicit expressions for $\mathbf{M}$ and $\mathbf{N}$ depend on the raw moment set and the corresponding central moment set, which will be discussed in the next subsection.

In the implementation of CLBM, the collision step is firstly executed in the central moment space. To be consistent with 
the central-moment-based collision operator, an external or internal force field $\mathbf{F}=\left[F_{x}, F_{y}, F_{z}\right]$ should be added by means of central moments [23]. By using a transformation method to incorporate forcing terms with a second-order trapezoidal scheme, the explicit form of collision step finally reads as follows [42]:

$$
\begin{aligned}
\left|\tilde{T}_{i}^{*}\right\rangle & =(\mathbf{I}-\mathbf{S})\left|\tilde{T}_{i}\right\rangle+\mathbf{S}\left|\tilde{T}_{i}^{\mathrm{eq}}\right\rangle+(\mathbf{I}-\mathbf{S} / 2)\left|C_{i}\right\rangle \\
& =(\mathbf{I}-\mathbf{S}) \mathbf{N M}\left|f_{i}\right\rangle+\mathbf{S N M}\left|f_{i}^{\mathrm{eq}}\right\rangle+(\mathbf{I}-\mathbf{S} / 2) \mathbf{N M}\left|R_{i}\right\rangle,
\end{aligned}
$$

where $\mathbf{I}$ is a unit matrix, $\mathbf{S}$ is the relaxation matrix, and $C_{i}$ and $R_{i}$ are forcing terms in central moment space and discrete velocity space, respectively. Due to the definitions of the transformation and shift matrices, both of them are invertible (explicit expressions for $\mathbf{M}^{-1}$ and $\mathbf{N}^{-1}$ can be easily obtained by software like MATLAB). The postcollision discrete DFs can be reconstructed by

$$
\left|f_{i}^{*}\right\rangle=\mathbf{M}^{-1}\left|T_{i}^{*}\right\rangle, \quad\left|T_{i}^{*}\right\rangle=\mathbf{N}^{-1}\left|\tilde{T}_{i}^{*}\right\rangle .
$$

In the streaming step, the postcollision discrete DFs in space $\mathbf{x}$ stream to their neighbors $\left(\mathbf{x}+\mathbf{e}_{i} \Delta t\right)$ along the characteristic lines as usual $[10,42]$,

$$
f_{i}\left(\mathbf{x}+\mathbf{e}_{i} \Delta t, t+\Delta t\right)=f_{i}^{*}(\mathbf{x}, t) .
$$

Then the fluid density $\rho$ and velocity $\mathbf{u}=\left[u_{x}, u_{y}, u_{z}\right]$ are updated by

$$
\rho=\sum_{i} f_{i}, \quad \rho \mathbf{u}=\sum_{i} f_{i} \mathbf{e}_{i}+\Delta t \mathbf{F} / 2 .
$$

From the above, it can be shown that when the shift matrix $\mathbf{N}$ is a unit matrix the CLBM degrades into an MRT-LBM on the specified raw moment set $\left|T_{i}\right\rangle$, and when all the relaxation parameters in the matrix $\mathbf{S}$ are equal to one another the CLBM degrades into an SRT-LBM. Thus we proclaim the above framework as a GMRT framework [42]. It can be also shown that the corresponding forcing scheme (given in the next subsection) can degrade into the MRT version and SRT version of the widely used forcing scheme by Guo et al. [44] under the corresponding conditions. Thus it is named as a consistent scheme [42].

\section{B. Central moment set, equilibria, and forcing terms}

In this subsection, we first discuss the central moment set, which is an important step to construct the CLBM. As discussed in Refs. [24,29], the conserved moments $\left(\tilde{k}_{000}, \tilde{k}_{100}\right.$, $\left.\tilde{k}_{010}, \tilde{k}_{001}\right)$ should be considered to represent the mass and momentum conservations, the second-order moments are chosen such that it allows correct representation of the momentum flux in the hydrodynamic equations, while the rest moments can be chosen order by order under a moments-independence constraint for a specified DVM. Premnath et al. adopted an orthogonal central moment set [24],

$$
\begin{aligned}
\left|\tilde{T}_{i}\right\rangle= & {\left[\tilde{k}_{000}, \tilde{k}_{100}, \tilde{k}_{010}, \tilde{k}_{001}, \tilde{k}_{110}, \tilde{k}_{101}, \tilde{k}_{011}, \tilde{k}_{200}-\tilde{k}_{020},\right.} \\
& \left(\tilde{k}_{200}+\tilde{k}_{020}+\tilde{k}_{002}\right)-3 \tilde{k}_{002},\left(\tilde{k}_{200}+\tilde{k}_{020}+\tilde{k}_{002}\right) \\
& -2 \tilde{k}_{000}, 3\left(\tilde{k}_{120}+\tilde{k}_{102}\right)-4 \tilde{k}_{100}, 3\left(\tilde{k}_{210}+\tilde{k}_{012}\right) \\
& -4 \tilde{k}_{010}, 3\left(\tilde{k}_{201}+\tilde{k}_{021}\right)-4 \tilde{k}_{001}, \tilde{k}_{120}-\tilde{k}_{102}, \tilde{k}_{210} \\
& -\tilde{k}_{012}, \tilde{k}_{201}-\tilde{k}_{021}, \tilde{k}_{111}, 3\left(\tilde{k}_{220}+\tilde{k}_{202}+\tilde{k}_{022}\right)
\end{aligned}
$$

$$
\begin{aligned}
& -4\left(\tilde{k}_{200}+\tilde{k}_{020}+\tilde{k}_{002}\right)+4 \tilde{k}_{000}, 3\left(\tilde{k}_{220}+\tilde{k}_{202}-2 \tilde{k}_{022}\right) \\
& -2\left(2 \tilde{k}_{200}-\tilde{k}_{020}-\tilde{k}_{002}\right), 3\left(\tilde{k}_{220}-\tilde{k}_{202}\right)-2\left(\tilde{k}_{020}\right. \\
& \left.-\tilde{k}_{002}\right), 3 \tilde{k}_{211}-2 \tilde{k}_{011}, 3 \tilde{k}_{121}-2 \tilde{k}_{101}, 3 \tilde{k}_{112} \\
& -2 \tilde{k}_{110}, 9 \tilde{k}_{122}-6\left(\tilde{k}_{120}+\tilde{k}_{102}\right) \\
& +4 \tilde{k}_{100}, 9 \tilde{k}_{212}-6\left(\tilde{k}_{210}+\tilde{k}_{012}\right)+4 \tilde{k}_{010}, 9 \tilde{k}_{221}-6\left(\tilde{k}_{201}\right. \\
& \left.+\tilde{k}_{021}\right)+4 \tilde{k}_{001}, 27 \tilde{k}_{222}-18\left(\tilde{k}_{220}+\tilde{k}_{202}+\tilde{k}_{022}\right) \\
& \left.+12\left(\tilde{k}_{200}+\tilde{k}_{020}+\tilde{k}_{002}\right)-8 \tilde{k}_{000}\right]^{\top} .
\end{aligned}
$$

According to the binomial theorem, a central moment can be expressed by raw moments from the lowest order to the same order [23,28], and thus the shift matrix $\mathbf{N}$ is a lower triangular matrix. As seen in Eq. (9), many combined terms are included in the orthogonal central moment set, which results in very tedious expressions in $\mathbf{N}$. In contrast, a nonorthogonal central moment set has been obtained in Ref. [40],

$$
\begin{aligned}
\left|\tilde{T}_{i}\right\rangle= & {\left[\tilde{k}_{000}, \tilde{k}_{100}, \tilde{k}_{010}, \tilde{k}_{001}, \tilde{k}_{110}, \tilde{k}_{101}, \tilde{k}_{011}, \tilde{k}_{200}\right.} \\
& -\tilde{k}_{020}, \tilde{k}_{200}-\tilde{k}_{002}, \tilde{k}_{200}+\tilde{k}_{020}+\tilde{k}_{002}, \tilde{k}_{120} \\
& +\tilde{k}_{102}, \tilde{k}_{210}+\tilde{k}_{012}, \tilde{k}_{201}+\tilde{k}_{021}, \tilde{k}_{120}-\tilde{k}_{102}, \tilde{k}_{210} \\
- & \tilde{k}_{012}, \tilde{k}_{201}-\tilde{k}_{021}, \tilde{k}_{111}, \tilde{k}_{220}+\tilde{k}_{202}+\tilde{k}_{022}, \\
& \tilde{k}_{220}+\tilde{k}_{202}-\tilde{k}_{022}, \tilde{k}_{220}-\tilde{k}_{202}, \tilde{k}_{211}, \tilde{k}_{121}, \\
& \left.\tilde{k}_{112}, \tilde{k}_{122}, \tilde{k}_{212}, \tilde{k}_{221}, \tilde{k}_{222}\right]^{\top} .
\end{aligned}
$$

Compared with Eq. (9), the expressions in Eq. (10) is simplified to some extent, but some combined terms still exist and the corresponding $\mathbf{N}$ is still tedious. In the present paper, we adopt the following central moment set,

$$
\begin{aligned}
\left|\tilde{T}_{i}\right\rangle=\left[\tilde{k}_{000}, \tilde{k}_{100}, \tilde{k}_{010}, \tilde{k}_{001}, \tilde{k}_{110}, \tilde{k}_{101}, \tilde{k}_{011}, \tilde{k}_{200},\right. \\
\\
\tilde{k}_{020}, \tilde{k}_{002}, \tilde{k}_{120}, \tilde{k}_{102}, \tilde{k}_{210}, \tilde{k}_{201}, \tilde{k}_{012}, \tilde{k}_{021}, \tilde{k}_{111}, \tilde{k}_{220}, \tilde{k}_{202}, \\
\left.\tilde{k}_{022}, \tilde{k}_{211}, \tilde{k}_{121}, \tilde{k}_{112}, \tilde{k}_{122}, \tilde{k}_{212}, \tilde{k}_{221}, \tilde{k}_{222}\right]^{\top},
\end{aligned}
$$

where the combined terms are completely eliminated. Correspondingly, the raw moment set is

$$
\begin{aligned}
\left|T_{i}\right\rangle=[ & k_{000}, k_{100}, k_{010}, k_{001}, k_{110}, k_{101}, k_{011}, k_{200}, k_{020}, k_{002}, k_{120}, \\
& k_{102}, k_{210}, k_{201}, k_{012}, k_{021}, k_{111}, k_{220}, k_{202}, k_{022}, k_{211}, k_{121}, \\
& \left.k_{112}, k_{122}, k_{212}, k_{221}, k_{222}\right] .
\end{aligned}
$$

As a result, the corresponding $\mathbf{N}$ is much simplified (see Appendix B). However, it should be noted that the normal stress differences $\left(k_{200}-k_{020}\right.$ and $\left.k_{200}-k_{002}\right)$ and the trace of the pressure tensor $k_{200}+k_{020}+k_{002}$ should be considered separately in CLBM [29], as do the corresponding central moments $\tilde{k}_{200}-\tilde{k}_{020}, \tilde{k}_{200}-\tilde{k}_{002}$, and $\tilde{k}_{200}+\tilde{k}_{020}+\tilde{k}_{002}$. To handle this problem, the widely used diagonal relaxation matrix can be modified slightly to be a block-diagonal matrix (similar method has been previously used for 2D CLBM [27,32,33]),

$$
\begin{array}{r}
\mathbf{S}=\operatorname{diag}\left\{s_{0}, s_{1}, s_{1}, s_{1}, s_{v}, s_{v}, s_{v},\left[\begin{array}{l}
s_{+}, s_{-}, s_{-} \\
s_{-}, s_{+}, s_{-} \\
s_{-}, s_{-}, s_{+}
\end{array}\right], s_{3}, s_{3}, s_{3},\right. \\
\left.s_{3}, s_{3}, s_{3}, s_{3 b}, s_{4}, s_{4}, s_{4}, s_{4 b}, s_{4 b}, s_{4 b}, s_{5}, s_{5}, s_{5}, s_{6}\right\},
\end{array}
$$


with $s_{+}=\left(s_{2 b}+2 s_{2}\right) / 3$ and $s_{-}=\left(s_{2 b}-s_{2}\right) / 3$. The kinematic and bulk viscosities are related to the relaxation parameters by $v=\left(1 / s_{2}-0.5\right) c_{s}^{2} \Delta t$ and $\xi=2 / 3\left(1 / s_{2 b}-0.5\right) c_{s}^{2} \Delta t$, respectively. Thus the transformation matrix $\mathbf{M}$ can be written explicitly according to Eqs. (4) and (12),

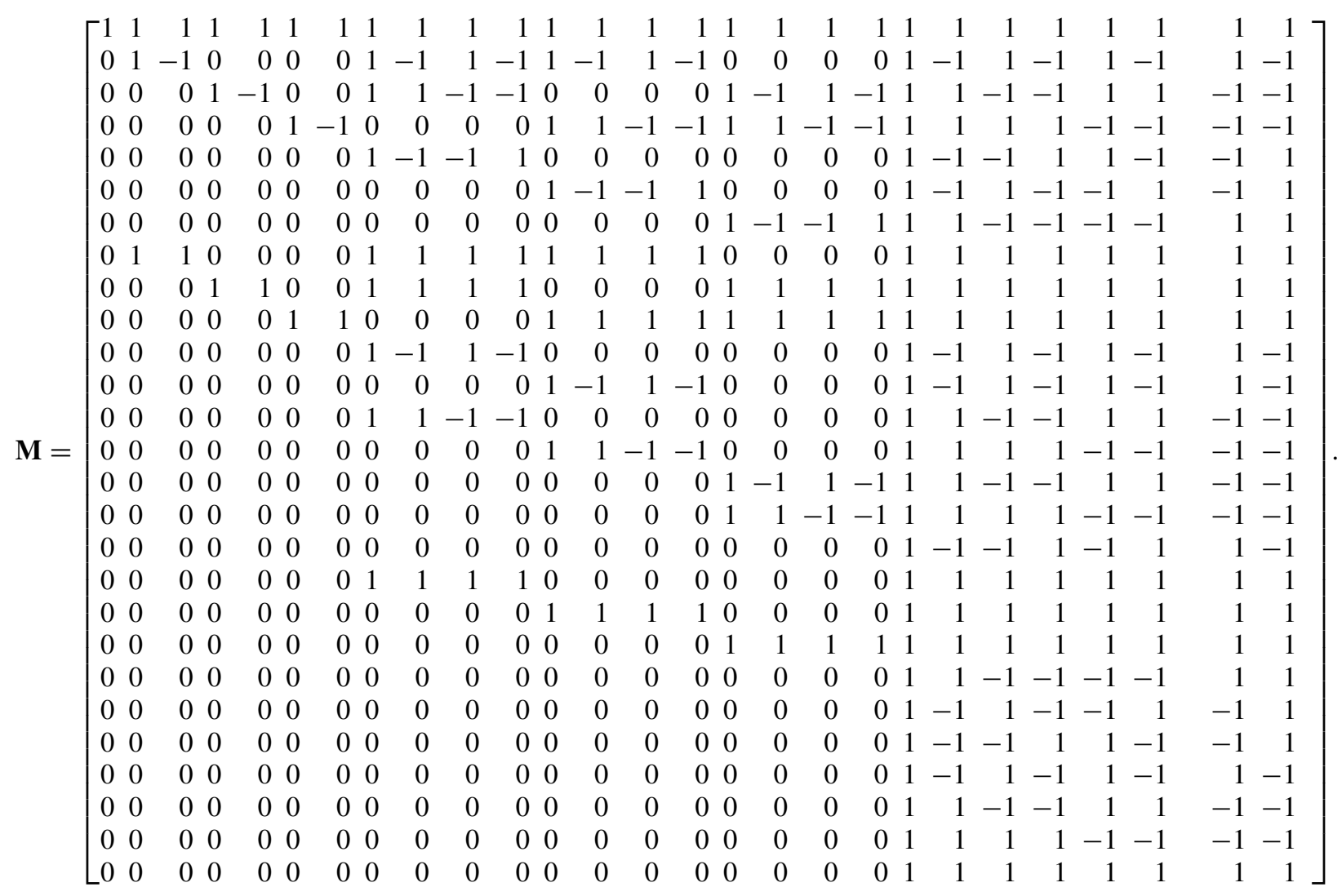

The shift matrix $\mathbf{N}$ can be obtained analogously according to the definition in Eq. (4). Taking the fifth row of $\mathbf{N}, \mathbf{N}_{4}$, as an example, the central moment $\tilde{T}_{4}$ can be expressed by raw moments from the lowest order to the same order,

$$
\begin{aligned}
\tilde{T}_{4} & =\left\langle f_{i} \mid\left(e_{i x}-u_{x}\right)\left(e_{i y}-u_{y}\right)\right\rangle \\
& =u_{x} u_{y} T_{0}-u_{y} T_{1}-u_{x} T_{2}+T_{4} .
\end{aligned}
$$

Thus $\mathbf{N}_{4}$ is written as

$$
\begin{gathered}
\mathbf{N}_{4}=\left[u_{x} u_{y},-u_{y},-u_{x}, 0,1,0,0,0,0,0,0,0,\right. \\
0,0,0,0,0,0,0,0,0,0,0,0,0,0,0] .
\end{gathered}
$$

The interested readers are kindly directed to the Supplemental Material [45] for the explicit expressions of $\mathbf{M}, \mathbf{N}, \mathbf{M}^{-1}$, and $\mathbf{N}^{-1}$.

To implement the collision step in Eq. (5), the equilibria and forcing terms in central moment space, $\left|\tilde{T}_{i}^{\mathrm{eq}}\right\rangle$ and $\left|C_{i}\right\rangle$, should be specified. In Refs. [40,41], the discrete EDFs [16], $f_{D, i}^{\mathrm{eq}}=\omega_{i} \rho\left[1+\left(\mathbf{e}_{i} \cdot \mathbf{u}\right) / c_{s}^{2}+\left(\mathbf{e}_{i} \cdot \mathbf{u}\right)^{2} / 2 c_{s}^{4}-\right.$ $\mathbf{u}^{2} / 2 c_{s}^{2}$, are adopted. However, we argue three points against this choice: (1) it results in a lot of velocity terms in $\left|\tilde{T}_{i}^{\mathrm{eq}}\right\rangle$ and $\left|C_{i}\right\rangle$, which is inconsistent with the physics of central moments; (2) it destroys the Galilean invariance for the off-diagonal elements of the third-order raw moments which are preserved naturally in the original CLBM by Geier et al. [22]; and (3) it leads to more computational cost for the calculation of $\left|\tilde{T}_{i}^{\text {eq }}\right\rangle$ and $\left|C_{i}\right\rangle$ compared with adopting the continuous equilibrium
DF. In this work, $\left|\tilde{T}_{i}^{\mathrm{eq}}\right\rangle$ are set equal to the continuous central moments of the continuous Maxwell-Boltzmann distribution $[22,23,27-29,42]$. Specifically, each element in $\left|\tilde{T}_{i}^{\text {eq }}\right\rangle$ is given as

$$
\begin{aligned}
k_{m n p}^{\mathrm{eq}}= & \left\langle f_{i}^{\mathrm{eq}} \mid\left(e_{i x}-u_{x}\right)^{m}\left(e_{i y}-u_{y}\right)^{n}\left(e_{i y}-u_{z}\right)^{p}\right\rangle \\
= & \int_{-\infty}^{+\infty} \int_{-\infty}^{+\infty} \int_{-\infty}^{+\infty} f^{M}\left(\xi_{x}-u_{x}\right)^{m}\left(\xi_{y}-u_{y}\right)^{n} \\
& \times\left(\xi_{z}-u_{z}\right)^{p} d \xi_{x} d \xi_{y} d \xi_{z},
\end{aligned}
$$

where $f^{M}$ is the continuous Maxwell-Boltzmann distribution in the continuous velocity space $\xi=\left[\xi_{x}, \xi_{y}, \xi_{z}\right]$,

$$
f^{M}(\rho, \mathbf{u}, \xi)=\frac{\rho}{2 \pi c_{s}^{2}} \exp \left[-\frac{(\xi-\mathbf{u})^{2}}{2 c_{s}^{2}}\right] .
$$

Substituting Eqs. (17) and (18) into the definition in Eq. (11). We can obtain

$$
\begin{gathered}
\left|\tilde{T}_{i}^{\mathrm{eq}}\right\rangle=\left[\rho, 0,0,0,0,0,0, \rho c_{s}^{2}, \rho c_{s}^{2}, \rho c_{s}^{2}, 0,0,0,0,0,0,0, \rho c_{s}^{4},\right. \\
\left.\rho c_{s}^{4}, \rho c_{s}^{4}, 0,0,0,0,0,0, \rho c_{s}^{6}\right]^{\top} .
\end{gathered}
$$

The corresponding discrete EDF is in fact a generalized local equilibrium $[23,27,31]$. In addition, when the viscosity is quite small, i.e., $v \sim O\left(10^{-7}\right)$, the higher-than-third-order central moment equilibria can be modified according to the factorized method $[24,46]$. Inspired by the approximation proposed by 
He et al. [47], the forcing effect in the Boltzmann equation can be given as

$$
R^{M} \approx-\frac{\mathbf{F}}{\rho} \cdot \nabla_{\xi} f^{M}=\frac{(\xi-\mathbf{u}) \cdot \mathbf{F}}{\rho c_{s}^{2}} f^{M} .
$$

Analogously, the forcing terms $\left|C_{i}\right\rangle$ are set equal to the continuous central moments of $R^{M}$ [42], i.e.,

$$
\begin{aligned}
\left|C_{i}\right\rangle= & {\left[0, F_{x}, F_{y}, F_{z}, 0,0,0,0,0,0, F_{x} c_{s}^{2}, F_{x} c_{s}^{2}, F_{y} c_{s}^{2}, F_{z} c_{s}^{2},\right.} \\
& \left.F_{y} c_{s}^{2}, F_{z} c_{s}^{2}, 0,0,0,0,0,0,0, F_{x} c_{s}^{4}, F_{y} c_{s}^{4}, F_{z} c_{s}^{4}, 0\right]^{\top} .
\end{aligned}
$$

Remarkably, the expressions for $\left|\tilde{T}_{i}^{\mathrm{eq}}\right\rangle$ and $\left|C_{i}\right\rangle$ are explicitly given in Eqs. (19) and (21), and thus the corresponding matrix manipulation is not needed in Eq. (5).

We now summarize the computational algorithm of the above proposed implementation and forcing scheme in 3D CLBM:

Step 1. Compute central moments $\left|\tilde{T}_{i}\right\rangle$ using the definition in Eq. (2). It should be noted that this method is quite basic and can be optimized by separating the transformation and shift substeps according to Eq. (4).

Step 2. Perform the collision step in Eq. (5).

Step 3. Reconstruct the postcollision raw moments according to $\left|T_{i}^{*}\right\rangle=\mathbf{N}^{-1}\left|\tilde{T}_{i}^{*}\right\rangle$, and reconstruct the postcollision DFs according to $\left|f_{i}^{*}\right\rangle=\mathbf{M}^{-1}\left|T_{i}^{*}\right\rangle$.

Step 4. Perform the streaming step and update the hydrodynamic variables according to Eqs. (7) and (8). Advance the time step and return to Step 1.

It is known that the most computationally demanding part in 3D CLBM is the reconstruction step of the postcollision DFs $[22,29,40]$. In the present work, the computational cost can be reduced significantly due to the facts that: first, the reconstruction step is divided into two substeps; second, the simplified central moment set is used such that the elements in $\mathbf{M}^{-1}$ and $\mathbf{N}^{-1}$ are much simplified (see Appendix B).

In addition, the present method can also be used to simplify the 3D MRT-LBM. For example, it can be found that the nonorthogonal transformation matrix $\mathbf{M}$ in Eq. (14) and its inverse matrix $\mathbf{M}^{-1}$ have 343 and 216 nonzero elements, respectively. In the orthogonal raw moment set by Premnath et al. [24], which has been used to construct the D3Q27 MRT-LBM in Ref. [48], both the $\mathbf{M}$ and $\mathbf{M}^{-1}$ have 416 nonzero elements. Therefore, compared with the D3Q27 MRT-LBM in Ref. [48], an MRT-LBM based on the present raw moment set can simplify the implementation and reduce the computational cost. As shown in Appendix C, the simulation results for 3D Lid-driven cavity flow by the nonorthogonal MRT-LBM are in good agreement with the benchmark solutions by Ku et al. [49], which implies that the nonorthogonal MRT-LBM can retain the numerical accuracy when simplifying the implementation.

\section{NUMERICAL SIMULATIONS}

In this section, several benchmark problems are conducted to verify the proposed 3D CLBM. In the simulation, the relaxation rates for the conserved central moments, $s_{0}$ and $s_{1}$ are set to 1.0. Unless otherwise specified, the tunable relaxation parameters for high-order central moments are also set to 1.0. The standard half-way bounce-back boundary scheme is used for wall boundaries.

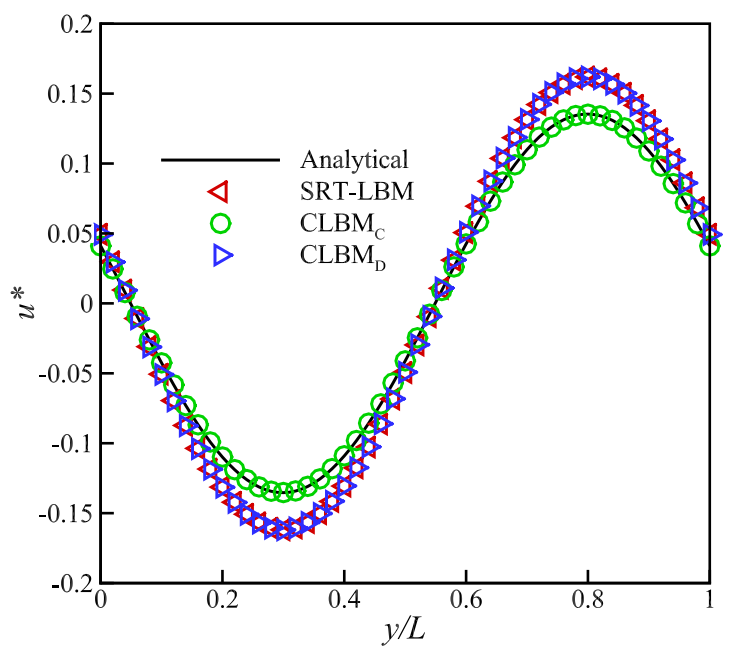

FIG. 1. Comparison of the velocity profiles for the decay of a shear wave at $t^{*}=2.0$ simulated by different methods.

\section{A. The decay of a shear wave}

First, the decay of a shear wave on a moving frame is considered. The initial conditions of the flow are given as

$$
\rho(0)=1.0, \quad \mathbf{u}=[A \sin (2 \pi / L), B, 0],
$$

where $A$ represents the initial amplitude of the shear wave, $B$ is the reference velocity component, and $L$ is the height of the computational domain. Periodic boundary conditions are used along the $x, y$, and $z$ axes, and the analytical velocity field is $u_{x}=A \sin [\phi(y-B t)] \exp \left(-\phi^{2} v t\right)$, where $\phi=2 \pi / L$. For the simulations, the amplitude is set to be $A=0.01$, the computational domain is covered by $5 \times 101 \times 5$ nodes.

First, we want to compare the central moment equilibria by Eq. (19) and by $f_{D, i}^{\mathrm{eq}}$, which are denoted by $\mathrm{CLBM}_{\mathrm{C}}$ and CLBM $_{\mathrm{D}}$, respectively. The SRT-LBM based on $f_{D, i}^{\mathrm{eq}}$ is also used for comparison. The profiles for the dimensionless velocity $u^{*}=u / A$ by different methods at the time $t^{*}=$ $\phi^{2} v t=2.0$ and Mach number $\mathrm{Ma}=B / c_{s}^{2}=0.3$ are shown in Fig. 1. It is found that the simulation result by $\mathrm{CLBM}_{\mathrm{C}}$ is in good agreement with the analytical solution, while there are visible differences between the numerical solutions by the other two methods and the analytical solution.

Then viscosity of the simulated fluid is obtained by measuring the time decay of the shear wave. The simulated viscosities at different $\mathrm{Ma}$ are compared in Fig. 2, while the original imposed viscosity is $v=0.05$. As is shown, the simulated viscosity by CLBM $_{C}$ is independent of the reference velocity (or Ma) and always agrees well with the imposed value. For the other two methods, the simulated viscosities decrease with the increase of the reference velocity. For example, the relative errors at $\mathrm{Ma}=0.3$ are $0.08 \%, 8.92 \%$, and $8.91 \%$ for $\mathrm{CLBM}_{\mathrm{C}}$, $\mathrm{CLBM}_{\mathrm{D}}$, and SRT-LBM, respectively.

The above results confirm our argument in Sec. IIB that using the discrete EDFs in CLBM as in Ref. [40] destroys the Galilean invariance (GI). It should be noted that there are two aspects to the issue of GI, one of which is related to the choice of the collision model and the other pertains to the choice of the discrete velocity model. For the standard lattice, the CLBM ${ }_{C}$ only preserves the GI naturally for the off-diagonal elements 


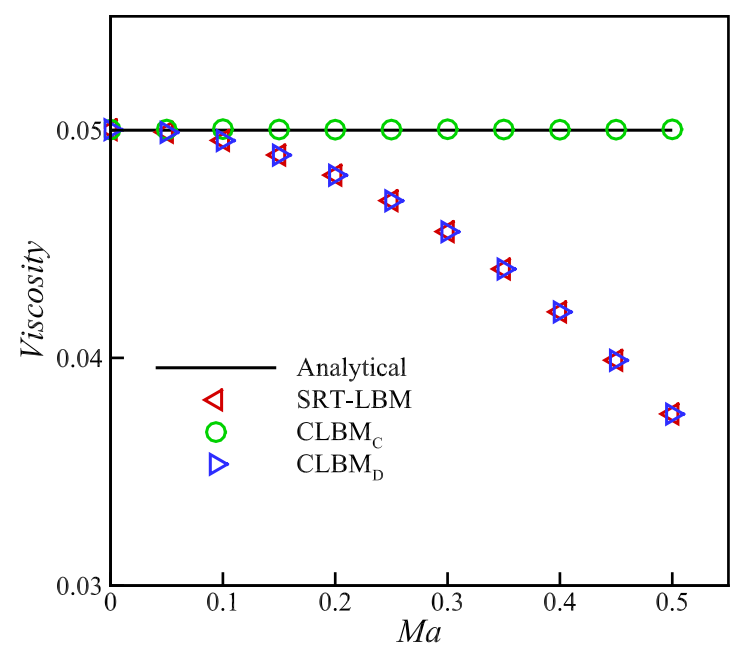

FIG. 2. Comparison of the simulated viscosities for the decay of a shear wave with Ma by different methods.

of the third-order raw moments. To restore the complete GI, additional correction terms [50] or more symmetrical lattice [51] are needed.

We now verify the computational efficiency of the present improved CLBM. We consider the same problem under different mesh sizes, i.e., $5 \times 101 \times 5,5 \times 201 \times 5$, and $5 \times 401 \times 5$, and measure the CPU time required for 10000 iterations by SRT-LBM, the present CLBM and the previous CLBM in Ref. [40]. For each case, the CPU time is the average value after removing the minimum and maximum in nine runs. The code is developed based on $\mathrm{C}++$ and runs on a laptop with Intel (R) Core (TM) i7-6500U CPU @ $2.5 \mathrm{GHz}$ and RAM $8.00 \mathrm{~GB}$. The implementation is basic for all the three models, without resorting to any optimization strategies, such as the preconditioning method [39] and the LAPACK library [40]. To be more specific, we use the program structure provided in the Appendix A in Ref. [6]. The collision step and streaming step are not combined into one loop, and different models are chosen by switching the collision subroutine in the main function. As shown in Table I, the CPU time for the present 3D CLBM is around 2.15 times of the one by SRT-LBM. Compared with the method in Ref. [40], where the computational cost overhead ratio is about 7.0, the present implementation shows significant reduction in the computational cost.

\section{B. Steady Poiseuille flow}

The second problem considered is a steady Poiseuille flow driven by a constant body force $\mathbf{F}=\left[F_{x}, 0,0\right]$. Thus the flow direction is along the positive direction of the $x$ axial. The

TABLE I. Comparison of the CPU time (s) required by SRT-LBM $\left(t_{S}\right)$, the present CLBM $\left(t_{C}\right)$, and the referenced CLBM $\left(t_{R}\right)$ in Ref. [40] for different mesh sizes.

\begin{tabular}{lccc}
\hline \hline Mesh & $t_{S}$ & $t_{C}$ & $t_{R}$ \\
\hline $5 \times 101 \times 5$ & 26.042 & 56.028 & 182.164 \\
$5 \times 201 \times 5$ & 51.728 & 111.838 & 361.184 \\
$5 \times 401 \times 5$ & 101.179 & 217.027 & 710.2165 \\
\hline \hline
\end{tabular}

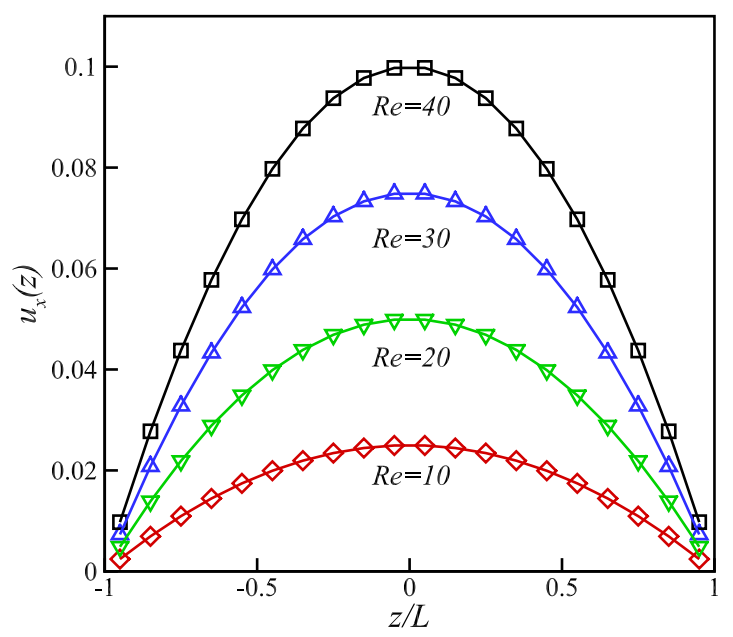

FIG. 3. Comparison between the numerical velocity profiles (symbols) and analytical solutions (solid lines) at $\operatorname{Re}=[10,20,30,40]$ for the steady Poiseuille flow.

analytical solution is $\mathbf{u}_{a}=\left[u_{x}, 0,0\right]$, where $u_{x}(z)=u_{0}(1-$ $\left.z^{2} / L^{2}\right)$ and $L$ is the half-height of the channel. The peak velocity is $u_{0}=F_{x} L^{2} / 2 v$, by which the Reynolds number is defined as $\operatorname{Re}=2 u_{0} L / v$. Due to the simple flow configuration, only five nodes are used to cover the length and width, and periodic boundary conditions are used in these two directions.

First, we choose the kinematic viscosity $v=0.1$, and 20 nodes are employed to cover the channel height $(2 L=20 \Delta x)$. The velocity profiles at a series of Reynolds numbers, $\mathrm{Re}=$ $[10,20,30,40]$, are plotted in Fig. 3. It can be seen in Fig. 3 that the simulation results are in very good agreement with the analytical solutions. As analyzed in previous studies $[19,43]$, when the relaxation rate for the energy flux is set to be $s_{3}=$ $\left(16-8 s_{2}\right) /\left(8-s_{2}\right)$, no numerical slip occurs in the steady Poiseuille flow for the MRT-LBM. It is shown recently in Ref. [42] that among the existing forcing schemes in twodimensional (2D) CLBM, only the consistent forcing scheme [42] preserves the above mentioned nonslip rule. We would like to stress that the consistent forcing scheme also has better performances in terms of accuracy and isotropy compared with the forcing scheme in Refs. [23,28,41]. For more information about the related analysis and discussion, interested readers are kindly directed to Ref. [42]. For the forcing scheme in 3D CLBM, we only consider the scheme proposed by Premnath et al. [24]. In their method, the orthogonal central moment set in Eq. (9) is adopted. In addition, their implementation is cumbersome [41] and is not based on the GMRT in this paper. Nevertheless, the principle of their forcing scheme is that only the first-order nonzero elements in $\left|C_{i}\right\rangle$ are perserved (see Eq. (26) in Ref. [24]). Using the present nonorthogonal central moment set, Eq. (21) should be correspondingly modified as

$$
\begin{aligned}
\left|C_{i}\right\rangle= & {\left[0, F_{x}, F_{y}, F_{z}, 0,0,0,0,0,0,0,0,0,0,0,\right.} \\
& 0,0,0,0,0,0,0,0,0,0,0,0]^{\top} .
\end{aligned}
$$

Here we choose two cases with $v=0.1$, and measure the global relative errors at different $s_{3}$. The global relative error is defined as

$$
E_{2}=\sqrt{\sum\left(\mathbf{u}-\mathbf{u}_{a}\right)^{2} / \sum \mathbf{u}_{a}^{2}}
$$




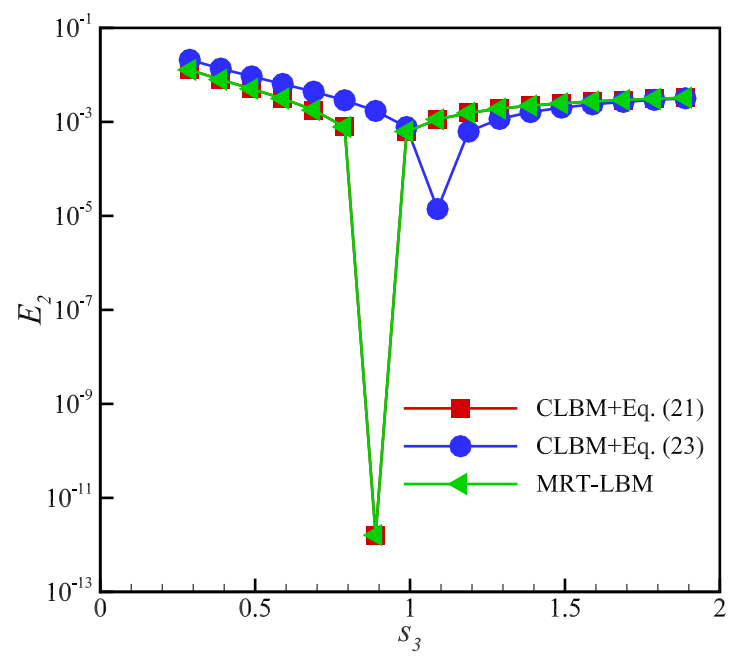

FIG. 4. Comparison of global relative errors by the present CLBM coupled with the forcing terms in Eqs. (21) and (23), and the MRTLBM [21,52] as a function of $s_{3}$ in steady Poiseuille flow at $s_{2}=5 / 4$. When the nonslip rule $s_{3}=\left(16-8 s_{2}\right) /\left(8-s_{2}\right)$ is satisfied, global relative errors by the present CLBM coupled the forcing scheme in Eq. (21) and the MRT-LBM approach almost zero.

where the summation operator is over all grid nodes. The D3Q19 MRT-LBM proposed in [21] coupled with the corresponding forcing scheme [52] is also adopted for comparison. It should be noted that the relaxation rate $s_{3}$ corresponds to the relaxation rate for the energy flux $\left(q_{a}\right.$ and $\left.m_{a}, a=x, y, z\right)$ in Ref. [21]. As shown in Fig. 4 when $s_{3}$ reaches the target value, $E_{2}$ by the present CLBM coupled the forcing scheme in Eq. (21) reduces significantly to a very small value, which is consistent with the result by the referenced MRT-LBM and confirms the consistent nonslip boundary condition in the present 3D CLBM. When the present CLBM is coupled with the forcing terms in Eq. (23) (actually the forcing scheme in Ref. [24]), the nonslip rule is not preserved. In the following simulations, the forcing terms in Eq. (21) are adopted.

We now proceed to test the validity of the modification method in Eq. (13) to separately relax the normal stress differences and trace of the pressure tensor. As shown in Fig. 5, we choose $v=0.1$ coupled with the nonslip rule and measure $E_{2}$ at different $\xi$. The variation trends by the present CLBM and the referenced MRT-LBM are basically the same. Thus the validity of the modification method in Eq. (13) is confirmed.

\section{Steady flow through a square duct}

We now consider the developed flow through a square duct by a driving force $\mathbf{F}=\left[F_{x}, 0,0\right]$, where the flow field is variable in both $y$ and $z$ directions. The flow has an analytical solution [53],

$$
\begin{aligned}
u_{x}(y, z)= & \frac{16 a^{2} F_{x}}{\rho \nu \pi^{3}} \sum_{n=1,3,5, \ldots}^{n=\infty}(-1)^{(n-1) / 2} \\
& \times\left[1-\frac{\cosh (n \pi z / 2 a)}{\cosh (n \pi / 2)}\right] \frac{\cos (n \pi y / 2 a)}{n^{3}},
\end{aligned}
$$

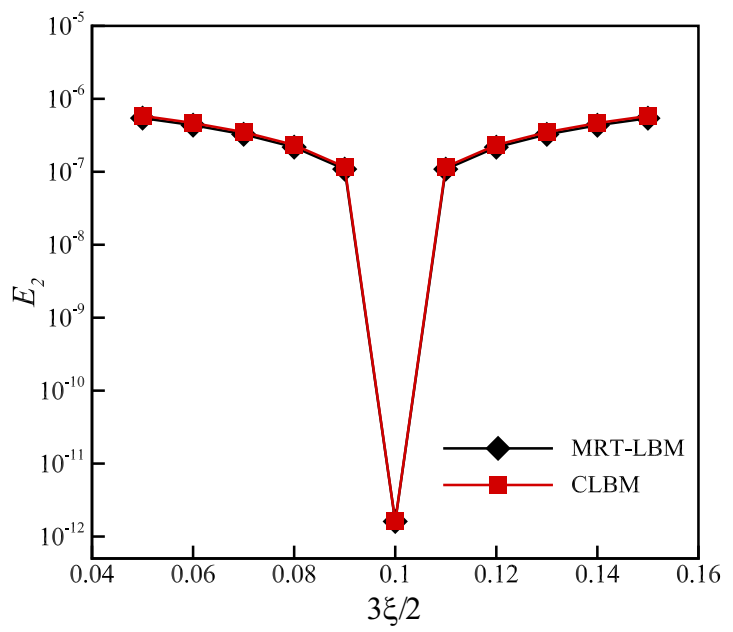

FIG. 5. Global relative error $E_{2}$ changes with the bulk viscosity $\xi$ in the present CLBM and the reference MRT-LBM in steady Poiseuille flow at $v=0.1$.

where $a$ is the duct half-width and $-a \leqslant y, z \leqslant a$. In the simulation, we set $F_{x}=2 \times 10^{-4}, v=0.2$, and $a=16 \Delta x$. The half-way bounce-back boundary condition is used at the walls, and thus the grid lines are located at $y=[-15.5 \Delta x, \ldots,-0.5 \Delta x, 0.5 \Delta x, \ldots, 15.5 \Delta x]$ and $z=[-15.5 \Delta x, \ldots,-0.5 \Delta x, 0.5 \Delta x, \ldots, 15.5 \Delta x]$. Only five nodes are used to cover the $x$ direction, along which the periodic boundary condition is adopted. The surface contour of the computed velocity field is shown in Fig. 6. It is seen that the present CLBM can reproduce the velocity distribution for steady flow through the square duct, and the simulation result is in qualitative agreement with the analytical solution.

To be quantitative, the simulation results for the velocity profiles at $z=[0.5 \Delta x, 7.5 \Delta x, 12.5 \Delta x]$ are compared with the analytical solutions in Fig. 7. It can be seen that the numerical results are in good agreement with the analytical solutions at different locations. In Sec. III B, we have found that the present CLBM holds the consistent nonslip boundary condition with the MRT-LBM. It should be noted that the derivation of the nonslip rule $s_{3}=\left(16-8 s_{2}\right) /\left(8-s_{2}\right)$ is based on the approximation that the velocity field varies in only one coordinate direction [43]. For the steady flow through a square duct, the velocity field varies in both $y$ and $z$ directions, thus the

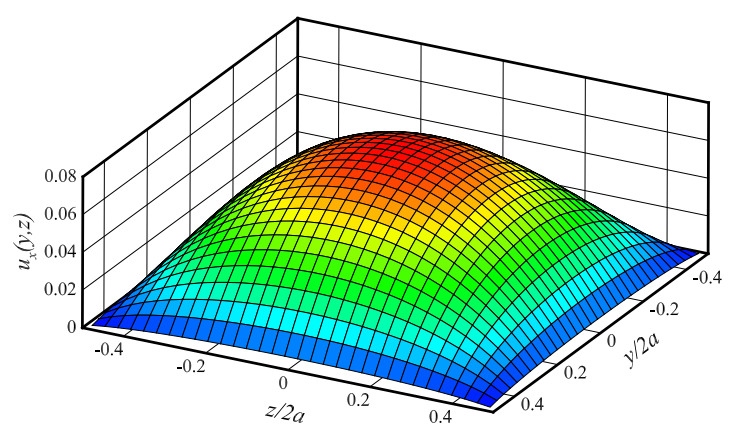

FIG. 6. Simulation result for the velocity distribution of steady flow through a square duct. The simulation parameters are $F_{x}=2 \times$ $10^{-4}, v=0.2$, and $a=16 \Delta x$. 


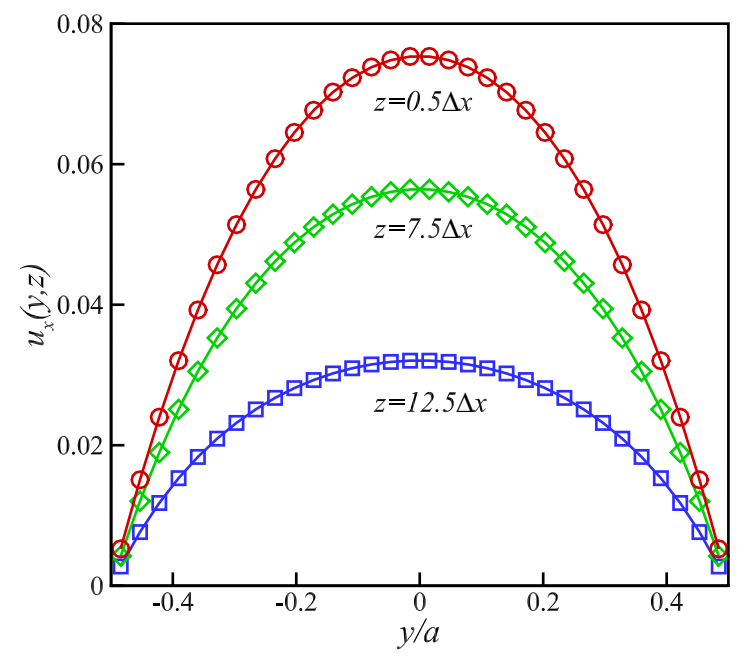

FIG. 7. Velocity profiles for steady flow through a square duct at $z=[0.5 \Delta x, 7.5 \Delta x, 12.5 \Delta x]$ (lines represent analytical solutions and symbols represent simulation results).

accurate nonslip condition may be not achieved. To verify our argument, we define the local relative error over the $z$ cross section,

$$
E(z)=\sqrt{\sum_{y}\left[u_{n x}(y, z)-u_{a x}(y, z)\right]^{2} / \sum_{y} u_{a x}(y, z)^{2}},
$$

where $u_{a x}$ and $u_{n x}$ denote the analytical and numerical velocities, respectively. Here we measure the change of $E(z)$ with $s_{3}$ at $z=[0.5 \Delta x, 7.5 \Delta x, 12.5 \Delta x]$. As shown in Fig. 8, when $s_{3}$ reaches the target value $1.33077, E(z)$ reduces to the smallest value for $z=0.5 \Delta x$ and $z=7.5 \Delta x$. However, the minimum point has a slight deviation from the target value for the case $z=12.5 \Delta x$. The reason for the deviation is that the $z=12.5 \Delta x$ cross section is near to the wall boundary, where the flow contains 2D feature and the unidirectional approximation is destroyed. Although our argument is presented for the present CLBM, it should also apply to the

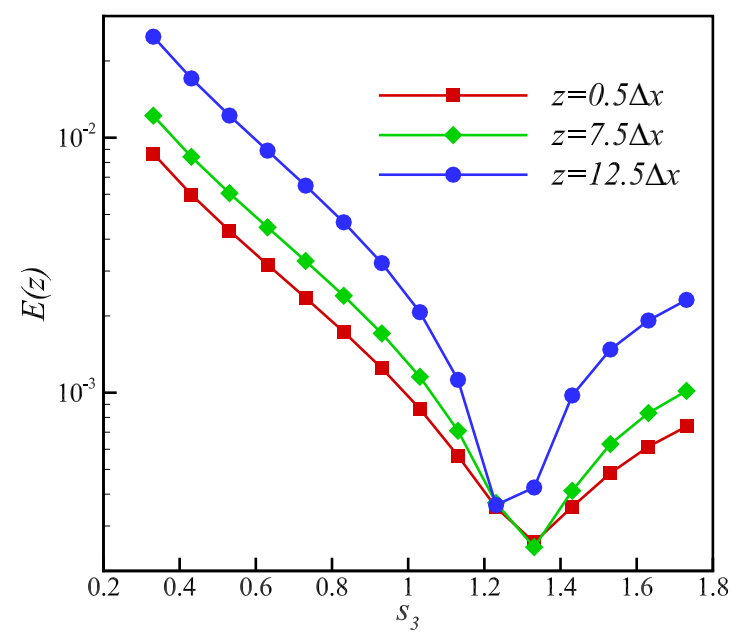

FIG. 8. Local relative error $E(z)$ for steady flow through a square duct changes with $s_{3}$ at $z=[0.5 \Delta x, 7.5 \Delta x, 12.5 \Delta x]$ (the kinematic viscosity is set to be $v=0.2$ ).

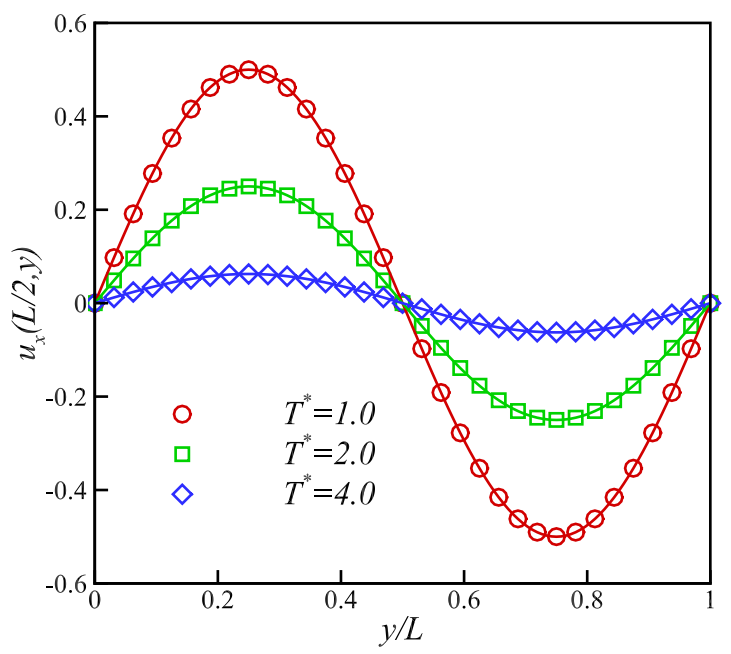

(a)

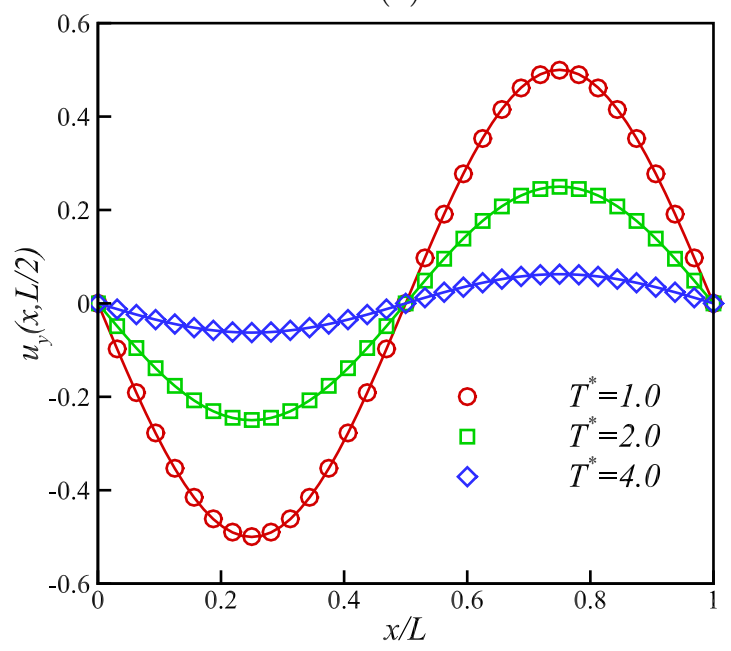

(b)

FIG. 9. Numerical results (symbols) and analytical solutions (solid lines) of the Taylor-Green vortex flow at $\operatorname{Re}=50$ and $T^{*}=$ [1.0,2.0,4.0]: (a) horizontal velocity profile in the $x=L / 2$ cross section and (b) vertical velocity profile in the $y=L / 2$ cross section.

MRT-LBM in general. As analyzed by Luo et al. [54] based on the 2D Lid-driven cavity flow simulation, the nonslip condition cannot be accurately satisfied for the complex flows, but usually a very good approximation can be obtained when using the nonslip rule $s_{3}=\left(16-8 s_{2}\right) /\left(8-s_{2}\right)$.

\section{Taylor-Green vortex flow}

As a final example, we want to test the present CLBM with the consistent forcing scheme for an unsteady flow where the force field depends on both time and space. The considered problem is the Taylor-Green vortex flow [55], which has an analytical solution

$$
\begin{aligned}
& u_{x}=-u_{0} \cos \left(k_{1} x\right) \sin \left(k_{2} y\right) \exp \left[-v\left(k_{1}^{2}+k_{2}^{2}\right) t\right], \\
& u_{y}=u_{0} \frac{k_{1}}{k_{2}} \sin \left(k_{1} x\right) \cos \left(k_{2} y\right) \exp \left[-v\left(k_{1}^{2}+k_{2}^{2}\right) t\right] .
\end{aligned}
$$

where $u_{0}$ is the amplitude of the imposed velocity field and $k_{1}$ and $k_{2}$ denote the wave numbers along the $x$ and $y$ directions. 


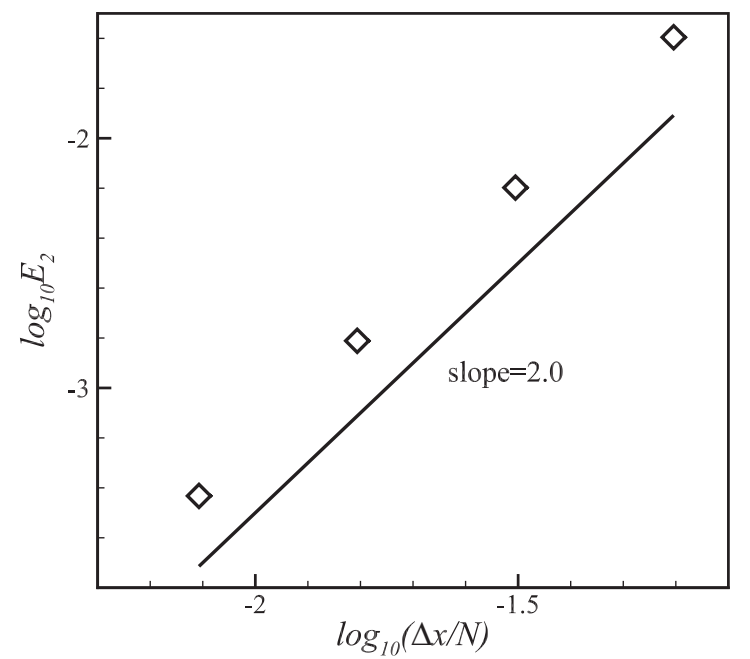

FIG. 10. Global relative error $E_{2}$ as a function of the grid spacing for the Taylor-Green vortex flow at $\operatorname{Re}=50$ and $T^{*}=4.0$. Symbols represent the measured global relative errors, and the fit slope of the results is 2.0345 .

The body force is given by

$$
\begin{aligned}
& F_{x}=-\left(\rho k_{1} u_{0}^{2} / 2\right) \sin \left(2 k_{1} x\right) \exp \left[-2 v\left(k_{1}^{2}+k_{2}^{2}\right) t\right], \\
& F_{y}=-\left(\rho k_{1}^{2} u_{0}^{2} / 2 k_{2}\right) \sin \left(2 k_{2} y\right) \exp \left[-2 v\left(k_{1}^{2}+k_{2}^{2}\right) t\right] .
\end{aligned}
$$

In the simulation, the computational domain is defined in $0 \leqslant$ $x, y \leqslant 2 \pi$, and covered by $L \times L$ grid points, while only five nodes are adopted along the $z$ direction. Thus the wave numbers are $k_{1}=k_{2}=2 \pi / L$. To eliminate the compressibility effect, $u_{0}$ is set to be 0.005 .

First, we choose $L=32 \Delta x$ and $v=0.0032$, and the corresponding Reynolds number $\operatorname{Re}=u_{0} L / v=50$. The numerical results of the horizontal velocity profile in the $x=L / 2$ cross section and the vertical velocity profile in the $y=L / 2$ cross section are plotted in Fig. 9, from which it can be seen that the numerical results are in good agreement with the analytical solutions at different nondimensional time, $T^{*}=$ $v\left(k_{1}^{2}+k_{2}^{2}\right) t / \ln 2$. Then simulations characterized by a series of grid resolutions are carried out, $L / \Delta x=[16,32,64,128]$, while the kinematic viscosity is obtained through $v=u_{0} L /$ Re. The log-log plot of the global relative error $E_{2}$ at $T^{*}=4.0$ as a function of the grid spacing is presented in Fig. 10, and the fit slope of the numerical results is 2.0345 . This demonstrates that the present CLBM with the consistent forcing scheme has second-order accuracy in space.

\section{CONCLUSIONS}

In this work, we present an efficient 3D CLBM formulation with a consistent forcing scheme for a general force field. In the method, the most computationally demanding reconstruction step in 3D CLBM is divided into two substeps based on the GMRT framework. A very simple central moment set is adopted to construct the cascaded operator such that the tedious combined terms in the shift matrix $\mathbf{N}$ are completely eliminated. To match the separate relaxations for certain second-order moments, the previously used diagonal relaxation matrix is modified to be a block-diagonal matrix.

Our proposed method is very efficient and easy to implement. Through the simulation of the decay of a shear wave, it is confirmed the present method can significantly reduce the computational cost compared with the recently proposed nonorthogonal CLBM [40]. The inconsistency of adopting the discrete EDF in the nonorthogonal CLBM [40] is revealed and discussed. Then the consistent forcing scheme [42] is verified by simulating several benchmark force-driven flows, highlighting very good properties of the developed methodology in terms of accuracy, convergence, and faithful implementation of the nonslip boundary condition.

The 3D CLBM developed is quite intelligible and general. Although the D3Q27 lattice is adopted for the derivation, the corresponding implementation in other lattices can be readily obtained. The new method puts the 3D MRT-LBM and CLBM into a unified general framework. Thus the developed methodology is not only applicable to CLBM but also can be adopted to simplify the 3D MRT-LBM, as demonstrated in Appendix C.

\section{ACKNOWLEDGMENTS}

Support from the MOST National Key Research and Development Programme (Project No. 2016YFB0600805) and the Center for Combustion Energy at Tsinghua University is gratefully acknowledged. Supercomputing time on ARCHER is provided by the UK Consortium on Mesoscale Engineering Sciences (UKCOMES) under the UK Engineering and Physical Sciences Research Council Grants No. EP/L00030X/1 and No. EP/R029598/1.

\section{APPENDIX A: D3Q19 CLBM}

For the D3Q19 lattice, the discrete velocities $\mathbf{e}_{i}=$ $\left[\left|e_{i x}\right\rangle,\left|e_{i y}\right\rangle,\left|e_{i z}\right\rangle\right](i=0,1, \ldots, 18)$ are defined as

$$
\begin{aligned}
\left|e_{i x}\right\rangle & =[0,1,-1,0,0,0,0,1,-1,1,-1,1,-1,1,-1,0,0,0,0]^{\top}, \\
\left|e_{i y}\right\rangle & =[0,0,0,1,-1,0,0,1,1,-1,-1,0,0,0,0,1,-1,1,-1]^{\top}, \\
\left|e_{i z}\right\rangle & =[0,0,0,0,0,1,-1,0,0,0,0,1,1,-1,-1,1,1,-1,-1]^{\top} .
\end{aligned}
$$

The central moment set can be extracted from Eq. (11)

$$
\left|\tilde{T}_{i}\right\rangle=\left[\tilde{k}_{000}, \tilde{k}_{100}, \tilde{k}_{010}, \tilde{k}_{001}, \tilde{k}_{110}, \tilde{k}_{101}, \tilde{k}_{011}, \tilde{k}_{200}, \tilde{k}_{020}, \tilde{k}_{002}, \tilde{k}_{120}, \tilde{k}_{102}, \tilde{k}_{210}, \tilde{k}_{201}, \tilde{k}_{012}, \tilde{k}_{021}, \tilde{k}_{220}, \tilde{k}_{202}, \tilde{k}_{022}\right]^{\top} \text {. }
$$

The block-diagonal matrix is given by

$$
\mathbf{S}=\operatorname{diag}\left\{s_{0}, s_{1}, s_{1}, s_{1}, s_{\nu}, s_{v}, s_{\nu},\left[\begin{array}{c}
s_{+}, s_{-}, s_{-} \\
s_{-}, s_{+}, s_{-} \\
s_{-}, s_{-}, s_{+}
\end{array}\right], s_{3}, s_{3}, s_{3}, s_{3}, s_{3}, s_{3}, s_{4}, s_{4}, s_{4}\right\} .
$$


The transformation matrix $\mathbf{M}$ can be written explicitly according to Eqs. (4) and (A2),

$$
\mathbf{M}=\left[\begin{array}{rrrrrrrrrrrrrrrrrrr}
1 & 1 & 1 & 1 & 1 & 1 & 1 & 1 & 1 & 1 & 1 & 1 & 1 & 1 & 1 & 1 & 1 & 1 & 1 \\
0 & 1 & -1 & 0 & 0 & 0 & 0 & 1 & -1 & 1 & -1 & 1 & -1 & 1 & -1 & 0 & 0 & 0 & 0 \\
0 & 0 & 0 & 1 & -1 & 0 & 0 & 1 & 1 & -1 & -1 & 0 & 0 & 0 & 0 & 1 & -1 & 1 & -1 \\
0 & 0 & 0 & 0 & 0 & 1 & -1 & 0 & 0 & 0 & 0 & 1 & 1 & -1 & -1 & 1 & 1 & -1 & -1 \\
0 & 0 & 0 & 0 & 0 & 0 & 0 & 1 & -1 & -1 & 1 & 0 & 0 & 0 & 0 & 0 & 0 & 0 & 0 \\
0 & 0 & 0 & 0 & 0 & 0 & 0 & 0 & 0 & 0 & 0 & 1 & -1 & -1 & 1 & 0 & 0 & 0 & 0 \\
0 & 0 & 0 & 0 & 0 & 0 & 0 & 0 & 0 & 0 & 0 & 0 & 0 & 0 & 0 & 1 & -1 & -1 & 1 \\
0 & 1 & 1 & 0 & 0 & 0 & 0 & 1 & 1 & 1 & 1 & 1 & 1 & 1 & 1 & 0 & 0 & 0 & 0 \\
0 & 0 & 0 & 1 & 1 & 0 & 0 & 1 & 1 & 1 & 1 & 0 & 0 & 0 & 0 & 1 & 1 & 1 & 1 \\
0 & 0 & 0 & 0 & 0 & 1 & 1 & 0 & 0 & 0 & 0 & 1 & 1 & 1 & 1 & 1 & 1 & 1 & 1 \\
0 & 0 & 0 & 0 & 0 & 0 & 0 & 1 & -1 & 1 & -1 & 0 & 0 & 0 & 0 & 0 & 0 & 0 & 0 \\
0 & 0 & 0 & 0 & 0 & 0 & 0 & 0 & 0 & 0 & 0 & 1 & -1 & 1 & -1 & 0 & 0 & 0 & 0 \\
0 & 0 & 0 & 0 & 0 & 0 & 0 & 1 & 1 & -1 & -1 & 0 & 0 & 0 & 0 & 0 & 0 & 0 & 0 \\
0 & 0 & 0 & 0 & 0 & 0 & 0 & 0 & 0 & 0 & 0 & 1 & 1 & -1 & -1 & 0 & 0 & 0 & 0 \\
0 & 0 & 0 & 0 & 0 & 0 & 0 & 0 & 0 & 0 & 0 & 0 & 0 & 0 & 0 & 1 & -1 & 1 & -1 \\
0 & 0 & 0 & 0 & 0 & 0 & 0 & 0 & 0 & 0 & 0 & 0 & 0 & 0 & 0 & 1 & 1 & -1 & -1 \\
0 & 0 & 0 & 0 & 0 & 0 & 0 & 1 & 1 & 1 & 1 & 0 & 0 & 0 & 0 & 0 & 0 & 0 & 0 \\
0 & 0 & 0 & 0 & 0 & 0 & 0 & 0 & 0 & 0 & 0 & 1 & 1 & 1 & 1 & 0 & 0 & 0 & 0 \\
0 & 0 & 0 & 0 & 0 & 0 & 0 & 0 & 0 & 0 & 0 & 0 & 0 & 0 & 0 & 1 & 1 & 1 & 1
\end{array}\right] .
$$

Thus the equilibria and forcing terms in the central moment space can be given as

$$
\left|\tilde{T}_{i}^{\mathrm{eq}}\right\rangle=\left[\rho, 0,0,0,0,0,0, \rho c_{s}^{2}, \rho c_{s}^{2}, \rho c_{s}^{2}, 0,0,0,0,0,0, \rho c_{s}^{4}, \rho c_{s}^{4}, \rho c_{s}^{4}\right]^{\top}
$$

and

$$
\left|C_{i}\right\rangle=\left[0, F_{x}, F_{y}, F_{z}, 0,0,0,0,0,0, F_{x} c_{s}^{2}, F_{x} c_{s}^{2}, F_{y} c_{s}^{2}, F_{z} c_{s}^{2}, F_{y} c_{s}^{2}, F_{z} c_{s}^{2}, 0,0,0\right]^{\top} .
$$

The explicit expressions of $\mathbf{M}, \mathbf{N}, \mathbf{M}^{-1}$, and $\mathbf{N}^{-1}$ for the D3Q19 CLBM are also provided in the Supplemental Material [45].

\section{APPENDIX B: SIMPLIFIED RECONSTRUCT STEP}

Intuitively, dividing the projection processes into two substeps in Eq. (6) may require more computational cost compared with the one step method. Actually, the computational cost is much reduced. Taking the D2Q9 CLBM as an example, it can be seen that the corresponding $\mathbf{M}^{-1}$ and $\mathbf{N}^{-1}$ have 39 and 40 nonzero elements, respectively. The elements in both $\mathbf{M}^{-1}$ and $\mathbf{N}^{-1}$ are very simple (see the Appendix in Ref. [42]). However, if the two substeps are combined into one step, then the reconstruction matrix $\mathbf{K}=\mathbf{M}^{-1} \mathbf{N}^{-1}$ is fully filled, and most of the elements are complex. Using the software MATLAB, it can be written row by row,

$$
\begin{aligned}
\mathbf{K}_{0}= & {\left[u_{x}^{2} u_{y}^{2}-u_{x}^{2}-u_{y}^{2}+1,2\left(u_{x} u_{y}^{2}-u_{x}\right), 2\left(u_{x}^{2} u_{y}-u_{y}\right),\left(u_{x}^{2}+u_{y}^{2}\right) / 2-1,\left(u_{y}^{2}-u_{x}^{2}\right) / 2,4 u_{x} u_{y}, 2 u_{y}, 2 u_{x}, 1\right], } \\
\mathbf{K}_{1}= & {\left[\left(-u_{x}^{2} u_{y}^{2}-u_{x}^{2} u_{y}+u_{x}^{2}+u_{x}\right) / 2,-u_{x} u_{y}^{2}-u_{x} u_{y}+u_{x}+1 / 2,-u_{x}^{2} u_{y}-u_{x}^{2} / 2,\right.} \\
& \left.-\left(u_{x}^{2}+u_{y}^{2}+u_{y}-1\right) / 4,\left(u_{x}^{2}-u_{y}^{2}-u_{y}+1\right) / 4,-u_{x}-2 u_{x} u_{y},-u_{y}-1 / 2,-u_{x},-1 / 2\right], \ldots, \\
\mathbf{K}_{8}= & {\left[\left(u_{x}^{2} u_{y}^{2}+u_{x}^{2} u_{y}-u_{x} u_{y}^{2}-u_{x} u_{y}\right) / 4,\left(u_{x} u_{y}+u_{x} u_{y}^{2}\right) / 2-\left(u_{y}+u_{y}^{2}\right) / 4,\left(u_{x}^{2} u_{y}-u_{x} u_{y}\right) / 2\right.} \\
& -\left(u_{x}-u_{x}^{2}\right) / 4,\left(u_{x}^{2}-u_{x}+u_{y}^{2}+u_{y}\right) / 8,\left(-u_{x}^{2}+u_{x}+u_{y}^{2}+u_{y}\right) / 8,\left(u_{x}-u_{y}\right) / 2+u_{x} u_{y} \\
& \left.-1 / 4, u_{y} / 2+1 / 4, u_{x} / 2-1 / 4,1 / 4\right] .
\end{aligned}
$$

It can be seen that the reconstruction step using the above $\mathbf{K}$ is cumbersome. For the 3D models, this effect is much amplified. As a result, the one-step method has higher computational cost than the two-step method.

Second, the simplified central moment set is used such that the filled numbers in $\mathbf{M}^{-1}$ are reduced and the elements in $\mathbf{N}^{-1}$ are much simplified. According to the relation between the raw moments and central moments, it is known that the shift matrix $\mathbf{N}$ is a lower triangular matrix. Due to the symmetry, the matrix $\mathbf{N}^{-1}$ is quite similar to $\mathbf{N}$, and the only difference is that signs for all the odd order velocity terms are opposite in the two matrices (see in the Supplemental Material [45]). For the three central moment sets in Eqs. (9)-(11), the first seven elements are the same. Therefore, the first seven rows in the corresponding shift matrices are the same and can be written as a $7 \times 27$ matrix,

$$
\mathbf{N}_{0-6}=\left[\begin{array}{rrrrrrrrrrrrrrrrrrrrrrrrrrr}
1 & 0 & 0 & 0 & 0 & 0 & 0 & 0 & 0 & 0 & 0 & 0 & 0 & 0 & 0 & 0 & 0 & 0 & 0 & 0 & 0 & 0 & 0 & 0 & 0 & 0 & 0 \\
-u_{x} & 1 & 0 & 0 & 0 & 0 & 0 & 0 & 0 & 0 & 0 & 0 & 0 & 0 & 0 & 0 & 0 & 0 & 0 & 0 & 0 & 0 & 0 & 0 & 0 & 0 & 0 \\
-u_{y} & 0 & 1 & 0 & 0 & 0 & 0 & 0 & 0 & 0 & 0 & 0 & 0 & 0 & 0 & 0 & 0 & 0 & 0 & 0 & 0 & 0 & 0 & 0 & 0 & 0 & 0 \\
-u_{z} & 0 & 0 & 1 & 0 & 0 & 0 & 0 & 0 & 0 & 0 & 0 & 0 & 0 & 0 & 0 & 0 & 0 & 0 & 0 & 0 & 0 & 0 & 0 & 0 & 0 & 0 \\
u_{x} u_{y} & -u_{y} & -u_{x} & 0 & 1 & 0 & 0 & 0 & 0 & 0 & 0 & 0 & 0 & 0 & 0 & 0 & 0 & 0 & 0 & 0 & 0 & 0 & 0 & 0 & 0 & 0 & 0 \\
u_{x} u_{z} & -u_{z} & 0 & -u_{x} & 0 & 1 & 0 & 0 & 0 & 0 & 0 & 0 & 0 & 0 & 0 & 0 & 0 & 0 & 0 & 0 & 0 & 0 & 0 & 0 & 0 & 0 & 0 \\
u_{y} u_{z} & 0 & -u_{z} & -u_{y} & 0 & 0 & 1 & 0 & 0 & 0 & 0 & 0 & 0 & 0 & 0 & 0 & 0 & 0 & 0 & 0 & 0 & 0 & 0 & 0 & 0 & 0 & 0
\end{array}\right] .
$$


From the eighth row, the difference appears. In the central moment sets in Eqs. (9) and (10), the eighth element is $\tilde{k}_{200}-\tilde{k}_{020}$, and the eighth row of $\mathbf{N}$ is written as

$$
\mathbf{N}_{7}=\left[u_{x}^{2}-u_{y}^{2},-2 u_{x}, 2 u_{y}, 0,0,0,0,1,0,0,0,0,0,0,0,0,0,0,0,0,0,0,0,0,0,0,0\right] .
$$

Differently, the eighth element in the present $\left|\tilde{T}_{i}\right\rangle$ is $\tilde{k}_{200}$, and the eighth row of $\mathbf{N}$ is written as

$$
\mathbf{N}_{7}=\left[u_{x}^{2},-2 u_{x}, 0,0,0,0,0,1,0,0,0,0,0,0,0,0,0,0,0,0,0,0,0,0,0,0,0\right] .
$$

It is seen that the formulation in Eq. (B4) is simpler than that in Eq. (B3). Obviously, this simplifying effect is more evident in the higher rows of $\mathbf{N}$. Therefore, compared with the two central moment sets in Eqs. (9) and (10), the present central moment set corresponds to a much simplified shift matrix.

\section{APPENDIX C: NONORTHOGONAL MRT-LBM FOR 3D LID-DRIVEN CAVITY FLOW}

In the D3Q27 nonorthogonal MRT-LBM, the raw moment equilibria are obtained by $\left|T_{i}^{\mathrm{eq}}\right\rangle=\mathbf{M}\left|f_{D, i}^{\mathrm{eq}}\right\rangle$. Although the force field is not considered in this case, the forcing terms in raw moment space can be directly obtained by $\left|G_{i}^{\mathrm{eq}}\right\rangle=\mathbf{M}\left|R_{G, i}\right\rangle$, where $R_{G, i}=\omega_{i}\left[\left(\mathbf{e}_{i}-\mathbf{u}\right) / c_{s}^{2}+\right.$ $\left.\left(\mathbf{e}_{i} \cdot \mathbf{u}\right) \mathbf{e}_{i} / c_{s}^{4}\right] \mathbf{F}$ refer to the forcing scheme by Guo et al. [44]. Analogously, a D3Q19 nonorthogonal MRT-LBM can be constructed using the transformation matrix $\mathbf{M}$ in Eq. (A4).

We now use the D3Q27 nonorthogonal MRT-LBM to simulate the 3D Lid-driven cavity. The flow is confined in a cubic box $L \times L \times L$ and driven by a top lid at $y=L$ with constant velocity $U=0.1$. The Reynolds number $\operatorname{Re}=$ $U L / v=400$ is considered in the simulation, and the length of the cubic box is set to be $L=64 \Delta x$. From Fig. 11, it can be seen that a pair of vortices are located near the bottom of the $x=0.5 \mathrm{~L}$ plane, which is consistent with the results reported in Ref. [49]. In addition, the velocity profiles by the nonorthogonal MRT-LBM are compared with the benchmark solutions [49] in Fig. 12. It can be seen the present simulation results are in good agreement

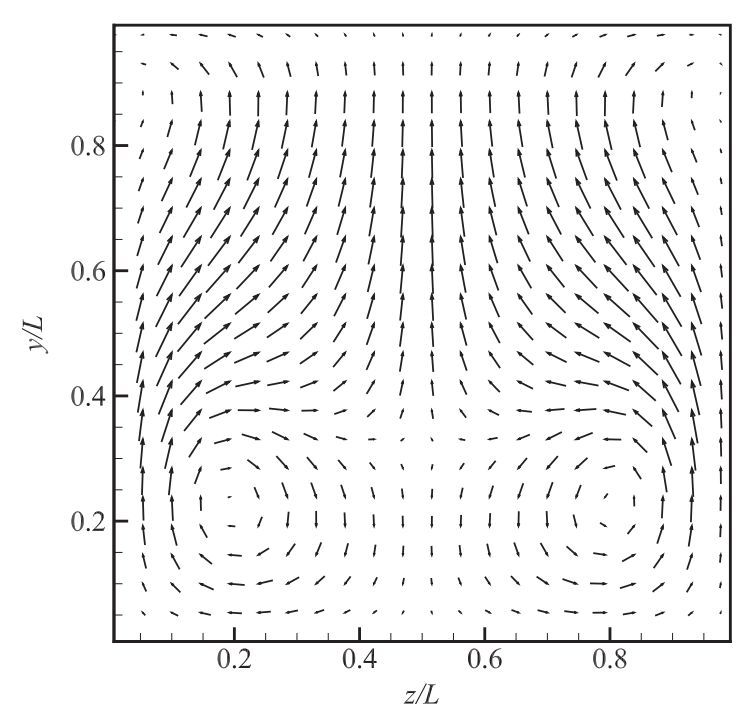

FIG. 11. The flow direction vector at $\operatorname{Re}=400$ in the $x=0.5 \mathrm{~L}$ plane. with the benchmark solutions. Furthermore, it is found that the nonorthogonal MRT-LBM requires approximately $25 \%$ less computational time than the orthogonal MRT model in Ref. [48].

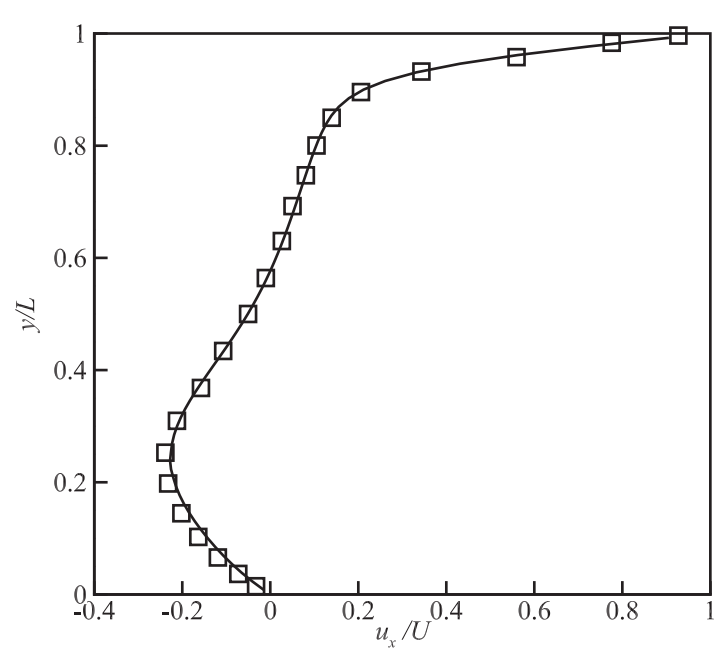

(a)

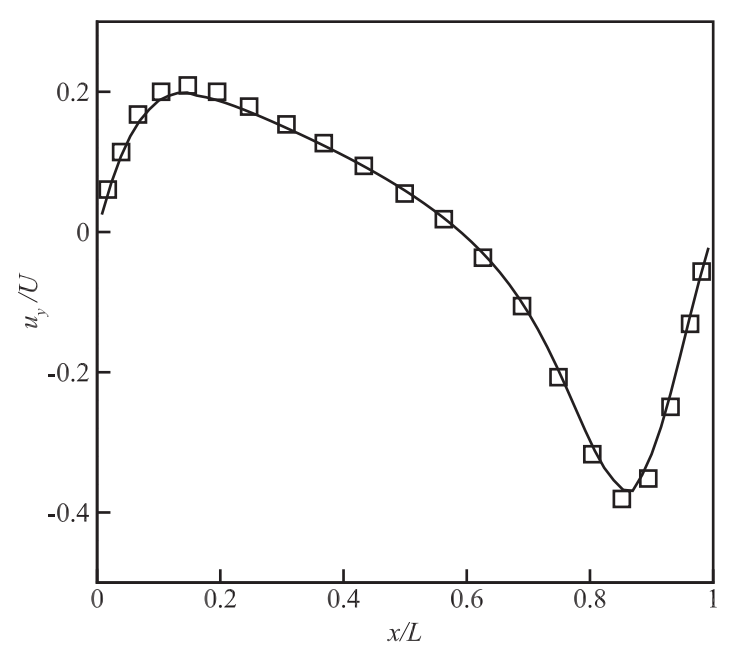

(b)

FIG. 12. The nonorthogonal MRT-LBM simulations (solid lines) and benchmark solutions [49] (symbols) of the 3D Lid-driven cavity flow at $\operatorname{Re}=400$ : (a) horizontal velocity profiles in the vertical centerline and (b) vertical velocity profile in the horizontal centerline. 
[1] Y.-H. Qian, S. Succi, and S. A. Orszag, Annual Reviews of Computational Physics III (World Scientific, 1995), pp. 195-242.

[2] S. Chen and G. D. Doolen, Annu. Rev. Fluid Mech. 30, 329 (1998).

[3] S. Succi, The Llattice Boltzmann Equation: For Fluid Dynamics and Beyond (Oxford University Press, Oxford, 2001).

[4] X. Shan and H. Chen, Phys. Rev. E 47, 1815 (1993).

[5] Y. Gan, A. Xu, G. Zhang, and Y. Li, Phys. Rev. E 83, 056704 (2011).

[6] Z. Guo and C. Shu, Lattice Boltzmann Method and Its Applications in Engineering, Vol. 3 (World Scientific, Singapore, 2013).

[7] Q. Li, Q. Kang, M. M. Francois, Y. He, and K. H. Luo, Int. J. Heat Mass Transf. 85, 787 (2015).

[8] Y. Gan, A. Xu, G. Zhang, and S. Succi, Soft Matter 11, 5336 (2015).

[9] A. Xu, T. Zhao, L. An, and L. Shi, Int.ernational J. Heat Fluid Flow 56, 261 (2015).

[10] Q. Li, K. H. Luo, Q. Kang, Y. He, Q. Chen, and Q. Liu, Prog. Energy Combust. Sci. 52, 62 (2016).

[11] A. Xu, T. Zhao, L. Shi, and X. Yan, Int. J. Heat Fluid Flow 62, 560 (2016).

[12] C. Lin, A. Xu, G. Zhang, and Y. Li, Combust. Flame 164, 137 (2016).

[13] W. Gong, Y. Zu, S. Chen, and Y. Yan, Sci. Bull. 62, 136 (2017).

[14] C. Lin, K. H. Luo, L. Fei, and S. Succi, Sci. Rep. 7, 14580 (2017).

[15] W. Gong, S. Chen, and Y. Yan, Int. Commun. Heat Mass Transfer 88, 136 (2017).

[16] Y. Qian, D. d'Humières, and P. Lallemand, Europhys. Lett. 17, 479 (1992).

[17] D. d'Humieres, Progress in Astronautics and Aeronautics 159, 450 (1994).

[18] P. Lallemand and L.-S. Luo, Phys. Rev. E 61, 6546 (2000).

[19] I. Ginzburg and D. d'Humières, Phys. Rev. E 68, 066614 (2003).

[20] C. Pan, L.-S. Luo, and C. T. Miller, Comput. Fluids 35, 898 (2006).

[21] D. d'Humières, Philos. Trans. Roy. Soc. Lond. A 360, 437 (2002).

[22] M. Geier, A. Greiner, and J. G. Korvink, Phys. Rev. E 73, 066705 (2006).

[23] K. N. Premnath and S. Banerjee, Phys. Rev. E 80, 036702 (2009).

[24] K. N. Premnath and S. Banerjee, J. Stat. Phys. 143, 747 (2011).

[25] H. Chen, P. Gopalakrishnan, and R. Zhang, Int. J. Mod. Phys. C 25, 1450046 (2014).

[26] M. Geier, A. Pasquali, and M. Schönherr, J. Comput. Phys. 348, 862 (2017).

[27] P. Asinari, Phys. Rev. E 78, 016701 (2008).
[28] D. Lycett-Brown and K. H. Luo, Comput. Math. Appl. 67, 350 (2014).

[29] D. Lycett-Brown, K. H. Luo, R. Liu, and P. Lv, Phys. Fluids 26, 023303 (2014).

[30] D. Lycett-Brown and K. H. Luo, Phys. Rev. E 94, 053313 (2016).

[31] L. Fei and K. H. Luo, arXiv:1610.07114.

[32] L. Fei, K. H. Luo, C. Lin, and Q. Li, Int. J. Heat Mass Transf. 120, 624 (2018).

[33] L. Fei and K. H. Luo, Comput. Fluids 165, 89 (2018).

[34] N. Shah, P. Dhar, S. K. Chinige, M. Geier, and A. Pattamatta, Numer. Heat Transfer B 72, 211 (2017).

[35] C. S. Kumar, S. Mohankumar, M. Geier, and A. Pattamatta, Int. J. Therm. Sci. 122, 201 (2017).

[36] K. V. Sharma, R. Straka, and F. W. Tavares, Int. J. Therm. Sci. 118, 259 (2017).

[37] A. De Rosis, Comput. Methods Appl. Mech. Eng. 319, 379 (2017).

[38] E. J. Falagkaris, D. M. Ingram, I. M. Viola, and K. Markakis, Computers \& Mathematics with Applications 74, 2348 (2017).

[39] F. Hajabdollahi and K. N. Premnath, Computers \& Mathematics with Applications (2017).

[40] A. De Rosis, Phys. Rev. E 95, 013310 (2017).

[41] A. De Rosis, Phys. Rev. E 95, 023311 (2017).

[42] L. Fei and K. H. Luo, Phys. Rev. E 96, 053307 (2017).

[43] Z. Guo and C. Zheng, Int. J. Comput. Fluid Dynam. 22, 465 (2008).

[44] Z. Guo, C. Zheng, and B. Shi, Phys. Rev. E 65, 046308 (2002).

[45] See Supplemental Material at http://link.aps.org/supplemental/ 10.1103/PhysRevE.97.053309 for the explicit expressions of M, $\mathrm{N}, \mathrm{M}^{-1}$ and $\mathrm{N}^{-1}$.

[46] M. Geier, A. Greiner, and J. Korvink, Eur. Phys. J. Spec. Top. 171, 55 (2009).

[47] X. He, S. Chen, and G. D. Doolen, J. Comput. Phys. 146, 282 (1998).

[48] K. Suga, Y. Kuwata, K. Takashima, and R. Chikasue, Comput. Math. Appl. 69, 518 (2015).

[49] H. C. Ku, R. S. Hirsh, and T. D. Taylor, J. Comput. Phys. 70, 439 (1987).

[50] N. I. Prasianakis, I. V. Karlin, J. Mantzaras, and K. B. Boulouchos, Phys. Rev. E 79, 066702 (2009).

[51] S. S. Chikatamarla and I. V. Karlin, Phys. Rev. Lett. 97, 190601 (2006).

[52] D. Zhang, K. Papadikis, and S. Gu, Int. J. Multiphase Flow 64, 11 (2014).

[53] F. M. White and I. Corfield, Viscous Fluid Flow, Vol. 3 (McGraw-Hill Higher Education, Boston, 2006).

[54] L.-S. Luo, W. Liao, X. Chen, Y. Peng, and W. Zhang, Phys. Rev. E 83, 056710 (2011).

[55] G. Taylor and A. Green, Proc. Roy. Soc. Lond. A 158, 499 (1937). 